\title{
28. COMMISSION DES GALAXIES
}

\section{Reports of Meetings}

President: B. Lindblad.

Secretary: G. C. McVittie.

The Commission met on 26, 27, 3I August and on I September. The proceedings consisted of $(a)$ the consideration of matters of business; $(b)$ a series of papers on the Magellanic Clouds; and $(c)$ a second series on the Evolution of Galaxies. A Joint Discussion on Radio Galaxies was held under the sponsorship of Commissions 28, 33, 34, $4^{\circ}$ and 43 .

\section{Business Meetings}

The President announced that the second part of Vorontsov-Velyaminov's Catalogue of Galaxies would appear shortly. Arp was also preparing a Catalogue of Peculiar and Interacting Galaxies. De Vaucouleurs announced that his reference Catalogue of Bright Galaxies (2600 objects) would be published in October 1964 by the University of Texas Press.

The Commission considered the proposal of the National Committee of Canada that the Hubble constant should be fixed at $100 \mathrm{~km} \mathrm{~s}^{-1} \mathrm{mpc}^{-1}$. It was resolved that this was premature but that authors should be asked to state clearly what value of the Hubble constant they chose to employ.

The Committee on the Magellanic Clouds was reorganized. S. C. B. Gascoigne had requested to be relieved of the Chairmanship and F. J. Kerr also wished to resign. The Committee was reconstituted as follows: A. D. Thackeray (Chairman), H. C. Arp, M. Duflot, Cecilia Payne-Gaposchkin, J. V. Hindman, J. Landi Dessy, E. M. Lindsay, P. Oosterhoff, B. Westerlund.

A letter from Dr Hodge drew attention to an identification atlas of the Clouds which he and Miss Wright had prepared, and expected to have printed before December. The atlas will consist of 80 pairs of charts in two colours, on which Harvard variables and other objects are identified on a scale of $16^{\prime \prime} / \mathrm{mm}$. Dr Thackeray pointed out that positions are not included, but gave a reminder about a table of positions of Harvard variables, copies of which are available on application to the Radcliffe Observatory.

Attention was called to the recent publication of IAU-URSI Symposium no. 20: 'The Galaxy and the Magellanic Clouds'.

The following Resolution was proposed by T. L. Page and G. J. Whitrow and was seconded by G. de Vaucouleurs:

'The members of Commission 28 and many others interested in external galaxies wish to compliment the President (Professor B. Lindblad) and the Secretary (Professor G. C. McVittie) for their preparation of the Draft Report for 1961-1964. From the confusion of uncertain observations and highly speculative theory they have drawn an orderly summary of 3 years progress that will stimulate further effective research. It is to be hoped that this high standard can be maintained in future years in spite of an earlier tradition that was less satisfactory.' The resolution was adopted.

G. de Vaucouleurs summarized the part of the Draft Report which referred to the Working Group on Galaxy Photometry. 
F. Zwicky reported on the work of the Committee on Supernovae in the following terms:

' 152 supernovae have been discovered and clearly identified as such from I 885 until I July 1964. The dates of discovery, the discoverers, the probable dates and apparent photographic magnitudes at maximum, as well as the spectroscopic types (I, II, III, IV and V) are listed in a report "List of supernovae discovered since I 885 " distributed to the members of the Committee. Copies of this report will be sent to interested members of the IAU upon request. Co-ordinates, structural types, apparent photographic magnitudes, NGC numbers and available symbolic velocities of recession of the galaxies in which supernovae appeared are also listed. After thorough discussion by the members of the Committee it is intended to reissue the list of supernovae as part of a monograph.

'To the information given in the Draft Report we add that two extragalactic supernovae of type $\mathrm{V}$ are now known ( $196 \mathrm{r} v$ in NGC $10_{5} 8$ and $1_{96} 6_{4} a$ in $\mathrm{NGC}_{3} 6_{3} \mathrm{r}$ ). Bertola from Asiago reports that the first of these, more than 3 years after maximum, brightened up one magnitude and is now (September 1964 ) at $m_{\mathrm{p}}=18 \cdot 0$. Spectra obtainable at this stage with large reflectors should be carefully examined because the date of first appearance of forbidden lines will allow us to estimate the mass of the ejected gas cloud.

'All data available for the construction of light curves of about 60 supernovae are being analysed by $Z$ wicky and his co-workers. Statistical analyses of the frequency of appearance of different types of supernovae in different types of galaxies are being carried out by Bertaud, Bertola and Zwicky.'

The membership of the Committee on Supernovae was reconstituted and was now to consist of the following: Ch. Bertaud (France); A. Boyarchuk (U.S.S.R.); L. Detre (Hungary); G. Haro (Mexico); B. J. Kukarkin (U.S.S.R.); H. Lambrecht (German Dem. Rep.); A. Meinel (U.S.A.); L. Rosino (Italy); J. L. Sersic (Argentina); A. D. Thackeray (U.K.); B. E. Westerlund (Australia); P. Wild (Switzerland); F. Zwicky, Chairman (U.S.A.).

The Draft Report was adopted by the Commission subject to minor editorial corrections.

The President proposed, and the Commission accepted, that the following list of names should be sent to the Executive Committee of the Union as those of persons who should form the Organizing Committee of the Commission: E. M. Burbidge, E. Holmberg, G. C. McVittie, B. Y. Mills, A. Sandage, A. D. Thackeray. The Executive Committee, at the final General Assembly on 3 September 1964 , made the following appointments: President: R. Minkowski. Vice-President: G. C. McVittie. Organizing Committee: E. M. Burbidge, E. Holmberg, B. Y. Mills, A. Sandage, A. D. Thackeray. The new President appointed E. Holmberg to be Secretary of the Commission.

The President (B. Lindblad) announced that the Executive Committee of the Union had under consideration a proposal to hold a Symposium before 1967 on the Evolution of Galaxies. The Commission resolved to endorse this proposal.

$G$. Contopoulos gave a brief account of IAU Symposium no. 25 .

\section{Scientific Meeting on the Magellanic Clouds}

Acting Chatrman: A. D. Thackeray.

SECRETARY: F. J. Kerr.

Cecilia Payne-Gaposchkin reported on a long investigation on 1146 cepheids and a number of other variables in the SMC from Harvard plates. The cepheid light curves show different forms, with a general grouping into asymmetric and sinusoidal types. The frequency distribution of periods is different for the two groups. The period-luminosity diagram shows a large dispersion, approximately $\mathbf{I}^{m}$. 
A. $\mathcal{F}$. Wesselink presented the results of Radcliffe observations of 45 SMC variables. The apparent limit is fainter than in the Harvard survey, but the stars are in more restricted regions. His frequency distribution has a maximum at a shorter period than that found by Mrs Gaposchkin. The possibility of absorption effects was discussed.

F. F. Kerr (for J. V. Hindman) discussed recent $2 \mathrm{I}-\mathrm{cm}$ observations of the SMC. Comparisons between $2 \mathrm{I}-\mathrm{cm}$ and $\mathrm{Ca}$ II velocities suggest that the two main masses, of which the Cloud appears to be composed, are moving away from each other in the line of sight, and that the stars are distributed between the two. There is general agreement in position between $\mathbf{H}_{\mathbf{I}}$ and blue giants and supergiants, but the density relationship is different in different regions. $H_{I}$ and $H$ II velocities agree closely in some cases, but not in others.

A. D. Thackeray (for M. W. Feast) reported on a new study of optical velocities in the LMC. A good rotation curve was found, with curvature in the outer parts; the centre of rotation is $\mathrm{I}^{\circ}$ to the north of the main bar. The new optical and radio velocities agree well. The small value of the velocity dispersion for both stars and nebulae (c. $10 \mathrm{~km} \mathrm{~s}^{-1}$ ) supports the idea of a flattened system. Ten possible planetary nebulae have been found in the LMC. Their velocities fit the rotation curve reasonably well.

$B$. Westerlund discussed two supernova remnants which he and Dr Mathewson have found in the LMC. These are the first two supernova remnants to be found in extragalactic systems. They are Type II (Population I) supernovae, and hence give information on metal abundance. The objects are $\mathrm{N}_{49}$ and $\mathrm{N} 63 \mathrm{~A}$, both contained in Constellation III, which has a large starrich arc. There may have been similar explosions in other regions. A proposal was made to account for the evolution and distribution of the present Population I in the LMC.

Ch. Fehrenbach: Les nombreux clichés obtenus avec le Grand Prisme Objectif de l'ESO ont permis à $\mathrm{Ch}$. Fehrenbach et Mme Duflot de détecter 230 étoiles membres, ou membres très probables, du Grand Nuage de Magellan. 200 de ces étoiles sont annoncées pour la première fois. Parmi les étoiles sont les membres les plus brillants du Grand Nuage (après S Dor).

A. Florsch a trouvé 30 membres du Petit Nuage. Ses recherches sont moins avancées. Ses clichés de vitesse radiale couvrent une zone de $8^{\circ} \times 6^{\circ}$ centrée sur le Petit Nuage de Magellan ainsi que quelques champs entre le Petit et le Grand Nuage.

A. D. Thackeray (for J. B. Hutchings) reported firstly on some $\mathrm{H}_{\gamma}$ studies in the Clouds. The relation between equivalent width and apparent magnitude fits the galactic curve for distance moduli of 18.8 and 18.65 for the LMC and SMC respectively.

Thackeray also reported that his coudé spectra of the two brightest supergiants show evidence of an expanding shell in each case. S Dor has recently faded to a record minimum (fainter than $\mathrm{II}^{m}$ ) and resembles $\eta$ Car in the spectrum. Probably both are slow supernovae.

\section{Scientific Meeting on the Evolution of Galaxies}

T. L. Page first urged that agenda be drafted for an IAU Symposium in 1965 , and noted that this topic is in about the same state of early development as the concepts of stellar evolution were in 1935. There is evidence of an evolutionary pattern in the many correlations between colour, mass, luminosity, dimensions, spectrum, radio emission, cluster membership and the morphological types recognized by Hubble. Ignoring the small percentage of peculiar galaxies (Q.S.O.'s, Seyfert galaxies, Vorontsov's interacting galaxies, dwarf irregulars, etc.) it can be assumed that this pattern is a result of differences in the initial conditions in gas clouds from which galaxies condensed: total mass, mean density, angular momentum, chemical composition, turbulence, magnetic fields, etc.

Page has measured the inclinations of lines in the spectra of over 50 galaxies, and has collected measurements by others on more than 70 . These data confirm Holmberg's correlation 
between mass-density and colour in the central regions of all types. They also show a trend (marred by large fluctuations) from higher total angular momentum in $\mathrm{Sb}$ and $\mathrm{Sc}$ types to lower values in $\mathrm{Sa}$, So and $\mathrm{E}$ types.

The most serious difficulty with this concept of galaxies condensing from gas clouds is in the large measured values of the mass-luminosity ratio (about 2 for spirals and 50 to 100 for elliptical galaxies). Using M. Schmidt's formula for the rate of star formation, Reddish's distribution for new-star masses, the mass-luminosity relation and currently accepted mainsequence life-times, Page and Hayes have calculated the mean $M / L$ and colour at ages up to ${ }_{10}{ }^{10}$ years, and noted the effect of varying the parameters. Measured values of mean $M / L$ and colour can be matched by these calculations only if small stars are formed in large numbers, with masses as small as O०OI solar mass.

Invisible mass, collapsed to densities above the Schwarzschild limit, might also account for this discrepancy. However, Bondi argued against this possibility on relativistic grounds and McVittie pointed out that very peculiar physical conditions were tacitly assumed in order to compress matter into the Schwarzschild limit. G. Burbidge favoured the collapse to this limit.

D. W. Sciama said that galaxy formation represents the first step in the chain of galactic evolution. To determine the mechanisms which are responsible for it, it is necessary to know the physical state of the gas out of which the galaxies have presumably formed. Recent observations place fairly close limits on the possible temperature of the gas, if its density is assumed to be around $10^{-29}-10^{-28} \mathrm{~g} \mathrm{~cm}^{-3}$, the range of values required by both the Einstein-de Sitter and steady state models of the universe. A lower limit on the temperature is imposed by $21 \mathrm{~cm}$ observations, which could have detected neutral atomic hydrogen in this density range-but did not. Thus the gas, if it exists, must be ionized and so its kinetic temperature presumably exceeds $10^{4}{ }^{\circ} \mathrm{K}$. On the other hand the observed cosmic X-ray background in the range $2-8 \AA$ places an upper limit of $4 \times 10^{6}{ }^{\circ} \mathrm{K}$ on the temperature. If future observations show that there is no sharp increase in this background out to $50 \AA$ (a limit set by the opacity of our Galaxy), then the temperature must be less than $4 \times 10^{5}{ }^{\circ} \mathrm{K}$.

Indirect arguments can be used to narrow down the possible temperature still further, although these arguments are not as decisive as the observational ones. Considerations of the heat input required to produce the estimated temperature, and of the thermal instability of the gas, both suggest that the actual temperature lies in the neighbourhood of $10^{5}{ }^{\circ} \mathrm{K}$.

At this temperature the intergalactic gas would be thermally unstable against the formation of single galaxies on a time-scale of about $10^{10}$ years. Thus this mechanism may be as important as gravitational instability for the formation of single galaxies. However galaxy clusters cannot be formed by thermal instability alone, and it seems likely that one will have to take detailed account of the features of particular cosmological models in order to devise fruitful theories of their origin.

W. H. McCrea commented that the thermal instability discussed by Sciama, as well as the gravitational instability analysed by Jeans, avoided the fundamental question: How did the universe attain the required unstable initial state? It was also necessary to remember that, if the steady-state theory was used, thermodynamic equilibrium could not be assumed in order to calculate degrees of ionization, etc. The way in which material was fed into the system had to be taken into account.

V. C. Reddish described calculations on some integrated properties of galaxies, calculations which were concerned with the relationships between the masses $M$, luminosities $L$ and interstellar gas masses $M_{\mathrm{gas}}$, and their dependence on the age $t_{1}$, the rate of star formation per unit mass of gas $\frac{\mathrm{d} N}{\mathrm{~d} t} / M_{\mathrm{gas}}=K$, and the lower mass limit of star formation $M_{1}$. 
An initial luminosity function had been obtained primarily from field stars in the Galaxy and the Magellanic Clouds with allowance for evolutionary effects. This led to a mass function, the number of stars per unit range of mass, $F(M) \propto M^{-2 \cdot 5+0 \cdot 3}$. The upper limit $M_{2}$ of this function had been put equal to $I 0 \circ M_{\odot}$ but the results did not depend on it significantly. They were however sensitive to the lower limit $M_{1}$. The mass density for the solar neighbourhood, determined by Oort, was satisfied by a value $M_{1}=0.027 M_{\odot}$.

Bright star counts in nearby galaxies for which $M_{\text {gas }}$ is known from $21 \mathrm{~cm}$ hydrogen line observations gave the rate of formation of massive stars per unit mass of gas; from this, the rate of formation of stars of all masses was obtained using the mass function. With allowance for the effect of mass ejection by supernovae, the time required for all but 2 per cent of the gas of the Galaxy to condense into stars was then found to be $1 \cdot 1 \times 10^{10}$ years, in reasonable agreement with the age of the oldest stars in the Galaxy.

The calculations for other galaxies were concerned primarily with relationships between $M / L$ and $M_{\text {gas }} / M$. The available observational data showed that $M / L$ increases as $M_{\text {gas }} / M$ decreases; however, when a galaxy is all gas and no stars, $M / L$ must again be very high. Therefore $M / L$ must have a minimum when plotted against $M_{\mathrm{gas}} / M$; this $\mathrm{V}$-shaped curve must be reproduced by the theoretical models.

The calculations of $M / L$ versus $M_{\text {gas }} / M$ had been carried out for four cases:

(I) $K$ varying; family of curves for various $M_{1} ; t_{1}$ constant;

(2) $M_{1}$ varying; family of curves for various $K$; $t_{1}$ constant;

(3) $t_{1}$ varying; $K$ and $M_{1}$ constant with the values used in the calculations for our own Galaxy;

(4) $K$ and $M_{1}$ both varying with $K \propto M_{1}^{-s}$; family of curves for various $s$; $t_{1}$ constant.

Cases $I$ and 2 gave curves completely contrary to the observed relationship and may be rejected as possible causes of the observed differences between galaxies.

Case 3 fits the observations well. Case 4 produces a family of curves of the right general shape for values of $s>1$; the curve with $s=1 \cdot 5$ fits the observations best and is closely similar to the curve of case 3 . Thus these cases cannot be distinguished on the $M / L$ versus $M_{\text {gas }} / M$ diagram, and their consequences are considered separately.

Case 3. $M / L, M^{\text {gas }} / M$ and $L / M^{\text {gas }}$ had been calculated as functions of age $t_{1}$, and the ages of galaxies for which observations gave one or more of these ratios had then been calculated. When arranged in order of increasing age, the galaxies group distinctly according to morphological type, from Irr through Sc and Sb to E. It does not necessarily follow that this is an evolutionary sequence; one may argue that in this region of space ellipticals were formed first, then $\mathrm{Sb}$, then $\mathrm{Sc}$ and most recently, irregulars.

Case 4. $s={ }_{1} \cdot 5$. With $K \propto M_{1}^{-1 \cdot 5}$ it could be shown that the variation in $K$ resulted only from changes in the rate of formation of those stars with masses lying within the range of variation of $M_{1}$. This condition would occur if stars were formed by fragmentation of a gas cloud, and if the mass function of the fragments was always the same but the mass of the smallest fragment that can condense to form a star varied from one galaxy to another.

Morton S. Roberts spoke on the properties of galaxies. The fairly systematic variation of colour, total mass, and relative neutral hydrogen content (as well as the ratio of these quantities to total luminosity) with structural type represent observational facts with which to test various theories of galactic evolution. Thus, any acceptable theory must not only account for the structural sequence of galaxies but also for the above related quantities.

We must account for the colour-magnitude relation displayed by the late-type spirals and irregulars as well as the increase in total mass and the decrease in the relative atomic hydrogen content as we go to earlier spirals. The systematic variation of the total mass to luminosity ratio also represents an observational result to be explained. 
It should be noted that with our present knowledge it is not possible to relate variations in the hydrogen content with the age of a galaxy, in terms of the present rate of star formation, because the conclusions of such an approach are so severely dependent on the assumptions, especially on the form of the total initial luminosity function for recent star formation.

$2 \mathrm{I}-\mathrm{cm}$ observations have greatly strengthened our knowledge of total mass estimates of galaxies. Recent 300-foot radio telescope measures have shown, however, that a new uncertainty exists in the interpretation of rotation curves (derived from inter-stellar material), namely, noncircular motions over significant regions of many extragalactic systems.

In discussing Compact Galaxies and the Compact Parts of Galaxies, F. Zwicky said that a search for the lowest energy states of cosmic matter had been conducted. In particular, numerous compact galaxies were discovered whose images were barely distinguishable from those of stars when photographed with the largest telescopes. The spectra of some compact galaxies are continuous, while others are of types $\mathrm{K}$ to $\mathrm{B}$, with or without emission lines. Great widths of the spectral lines indicate high internal motions. Indicative absolute photographic magnitudes found so far lie in the range - $16 \cdot 0$ to $-20 \cdot 0$, masses are ${ }_{10}^{10} M_{\odot}$ to $10^{12} M_{\odot}$, diameters $100 \mathrm{pc}$ to $3000 \mathrm{pc}$ and indicative relative mass-luminosity ratios from 50 to 200 . Some compact galaxies show normal colours, others have $U-B, B-V$ similar to white dwarfs and radio sources with quasi-stellar nuclei. In high galactic latitudes about one compact galaxy brighter than $m_{\mathrm{p}}=17.0$ per two square degrees was found. A list of 200 prospective objects was being distributed at the XIIth General Assembly. Although generally stable, compact galaxies may implode and explode as a consequence of the ignition of various chain reactions and thus temporarily reach high visual and radio luminosities. Our search for afterstages of such explosions led to the discovery of numerous objects of unusual shapes, spectra and polarization. These exploded galaxies are being tested for radio-emission.

Some important aspects of the new discoveries are; $a$ ) Compact galaxies represent vital stages in the evolution of galaxies; $b$ ) Compact galaxies may add materially to the average density of matter in the universe; $c$ ) Compact galaxies will enable us to test decisively Einstein's prediction of the bending of light through gravitational fields. If successful, the results of these tests can be used to establish an absolute distance scale.

The subject of continual creation and the formation of galaxies was dealt with by $W . H$. McCrea who spoke as follows:

'I suggest that we should consider the possibility that, if there is a process of continual creation, it depends essentially upon already-existing matter, instead of being almost independent of such matter as hitherto supposed. In fact, I consider the hypotheses: (A) There is a process of interaction of matter with matter in which matter is not strictly conserved, so that all matter is the potential producer of new matter. (B) A galaxy grows in accordance with (A) until some limiting condition sets in.

'We picture an expanding universe of galaxies in which some galaxies from time to time eject fragments of material. According to (A) each such fragment is the potential producer of new matter; if it is successful as such, then it is the embryo of a new galaxy. According to (B), this grows until the limiting condition is reached; this might be fragmentation into stars.

'The idea is speculative but is less so than the sort of continual creation previously considered, for the required (nuclear) reaction would be an ordinary physical process and not truly "creation". Also it is more economical for, besides achieving what the previous theory did, it accounts also for the concentration of matter into galaxies.

'There is recent evidence that violent events in galaxies do eject matter; had this evidence been available when continual creation was proposed, it is not unlikely that the process would even then have been considered to depend upon already-existing matter. On the other hand, 
there is no evidence that nature does set itself the task of forming galaxies out of matter distributed more or less uniformly through space.

'The theory would allow galaxies to reach maturity quickly or slowly. If quickly, it would be rare to see an immature galaxy; this situation would be compatible with the existence of the sort of sequence discussed by Holmberg.

'Hypothesis (A) would require matter to produce predominantly matter, and not anti-matter; otherwise the process would be self-defeating. By symmetry, anti-matter would have to produce predominantly anti-matter. So if at any epoch the expanding universe were composed of patches of matter and patches of anti-matter, then each patch would proceed to grow indefinitely; ultimately, any observer would see only one sort of matter (which for him would be ordinary matter). Such considerations show that the theory may give a rational explanation as to why the universe appears to be composed of one kind of matter only, this having long been an unsolved problem. More, generally, if we have a universe that is expanding and selfreproducing, we may begin to understand why it should be approximately the same everywhere that we can see.'

$M$. Laffineur contributed the following remarks regarding quasi-stellar radio sources:

'The identification of several radio sources with optical objects of stellar appearance has raised quite a few new problems for astronomers: These "stars" seem to have a high radial velocity according to the shift of their emission lines, their spectra show the Balmer series of hydrogen, some metallic lines and some forbidden lines but how can we explain their positive radial velocity shown to be as large as half the velocity of light?

'For the small number of very well observed objects, the positive velocity suggests a recession movement. If we apply Hubble's law of the expansion of the universe the distance we would find amounts to more than one billion light years. With this distance in mind we deduce for the intrinsic luminosity an extremely high value, much greater than that of the average galaxies; furthermore the small apparent diameter leads us to assume a highly condensed object having a few hundreds parsecs in diameter (versus more than 20000 p.c. for our own Galaxy). Unexpectedly the magnitude of that powerful galaxy varies rapidly up or down $0^{m} \cdot 4$ in less than I year.

'Two hypotheses dealing with nearby stars have been discarded, the first being a strong radioemissive star moving with relativistic speed, the second an ultra dense star affected by gravity reddening.

'We suggest another hypothesis: why would those objects not be nearby stars with normal proper motions, emitting very fast jets of neutral plasma, jets that would appear to us as steady as the flame of a giant blow-torch. When the matter in the jets recombines it would become brighter than the star itself and the spectrum would show the velocity of the ejected matter relative to the observer. The shift of the optical spectrum would thus be easily explained as well as the generation of strong radio waves, the variability at short periods and the existence of complex radio sources.

'Of course there is one objection: why do we see only, up to now, recession velocities and reddened spectra? The directions of the jets should be at random as regards the Earth! This is indeed a serious objection but the number of well observed objects is very small and the randomness applies only to large numbers. In addition our atmosphere prevents recording very high approach velocities, pushing all the well known spectral lines into the unobservable ultraviolet. The answer can only come from further observations, possibly in spectral regions including the rocket UV.'

$R$. D. Davies described a high resolution survey of neutral hydrogen in $\mathrm{M}_{3} \mathrm{I}$ carried out at Jodrell Bank by himself, S. T. Gottesman and V. C. Reddish. The survey had been made with 
a $15^{\prime} \times 15^{\prime}$ beam and a $40 \mathrm{~km} / \mathrm{s}$ bandwidth. Neutral hydrogen was found out to $2.5^{\circ}$ along the major axis from the centre; it was most strongly concentrated in a ring ro kpc from the centre. The rotation curves of the northern and southern areas of the galaxy show significant differences of up to $20 \mathrm{~km} / \mathrm{s}$. An estimate of the total mass derived from the rotation curve gives a value of $1.4 \times 10^{11}$ solar masses out to $2.5^{\circ}$ from the centre while the neutral hydrogen mass is $2.4 \times 10^{9}$ solar masses out to this distance.

M. F. Walker spoke on the subjects of composite photographs of $\mathrm{M}_{33}$ and on NGC ro68. The composite photographs were produced from negatives taken with the I20-inch reflector at Lick in blue and infrared light. They brought out the following features:

(A) The elliptical smooth distribution of (resolved) Population II red giants. This distribution reminds us that the dynamics of a Sc galaxy are determined by this smoothly distributed population and not by the more spectacular spiral arms, and suggests that if star formation in the arms is not continuous, then, at times, M33 might resemble a (dusty) elliptical galaxy.

(B) The M-type supergiants tend to cluster with the clouds of OB stars in the star clouds in the spiral arms. These aggregates appear to be very similar to the $h$ and $\chi$ Perseus aggregate in our own Galaxy.

(C) There is a tendency for a deficiency of M-type supergiants to occur in those star clouds nearest the centre of the galaxy. It is not clear whether this is an age effect but whatever it is, it is not a unique function of radial distance since NGC 604, far out from the centre, also has no $\mathrm{M}$ supergiants associated with it; this object, containing both OB stars and nebulosity is presumably somewhat younger than the other star clouds in the spiral arms.

With regard to NGC 1068, Walker said that spectroscopic observations of this Seyfert galaxy had been obtained using the prime-focus spectrograph and the Lallemand electronic camera attached to the coudé spectrograph of the I20-inch reflector. Observations have been made in a number of position angles. These show:

(A) The existence in the bright, semi-stellar nucleus of at least 4 clouds of gas which are being ejected with velocities up to about $800 \mathrm{~km} / \mathrm{s}$ and internal turbulent velocities of about $600 \mathrm{~km} / \mathrm{s}$. These clouds occur in a region of angular diameter of about $6^{\prime \prime}$ to $8^{\prime \prime}$ and the region agrees with the observed size of the radio source in this system.

(B) The energy curve of the semi-stellar nucleus is flatter than that of $\mathrm{M}_{32}$, and is nearly constant between $3500 \AA$ and $3900 \AA$. Photoelectric measurements of polarization in the nucleus have been made in yellow and ultraviolet light. These show little or no polarization in the yellow, 13 per cent in the ultraviolet light, the position angle of electric vector being about $90^{\circ}$. Thus, there may be synchrotron radiation in the nucleus, obscured by radiation from late-type stars in yellow light, and becoming detectable in the UV.

(C) Outside the nucleus, the emission and absorption lines show that, within about $25^{\prime \prime}$ of the nucleus, non-circular motions occur, and that a difference between the motions of the stars and the gas exists. These disturbances may be related to the violent events occurring in the nucleus.

The relativistic morphology of elliptical galaxies was the subject of a paper by $D . G . B$. Edelen. A class of kinematical models of galactic structures had been constructed under the assumption that a galaxy had a definite physical boundary that is geometrically stable with respect to the Einstein gravitational field. No assumptions were made concerning the form of the momentum-energy tensor in the interior or the exterior of a galaxy other than those demanded by the requirement that the Einstein field equations be soluble in the presence of boundary surface jump strengths in some component of the momentum-energy tensor. The results of previous studies in the dynamics of discontinuity (boundary) surfaces in general relativity are thus applicable. It was shown that there must exist a function $\psi$ on the bounding 
surface that satisfies the surface Klein-Gordon equation. This function, in the form $\exp (-\psi)$, gives the modulus of the vector field that generates the time-oriented irrotational isometry of the intrinsic surface geometry as demanded by the requirement of strong geometric stability. Solutions of the surface equations on general quadrics led to equilibrium contours for the class of models. In the linearized case, in which $\psi$ depended only on the colatitude angle on the surface of an unperturbed oblate spheroid, a simple algorithm could be given: (I) determine a longitude independent, single-valued bounded function $\psi$ on the surface of the oblate spheroid in Euclidean three-space that satisfies the Helmholtz equation $\Delta \psi+\xi \psi=0$ for $\xi>0$; (2) if the equations of the surface of the oblate spheroid are $x^{i}=f^{i}(\alpha, \beta)$, where $\alpha$ and $\beta$ are the colatitude and longitude respectively, then the equations of the equilibrium boundary of a galaxy are given by $\bar{x}^{i}=f^{i}(\alpha, \beta) \exp (-\psi(\alpha))$. The shapes obtained in this way provided a morphological set having detailed correspondence to the optical morphology of the ellipticals (and possibly the SO's) in the real world. In particular, the observed boundaries of such disparate types as NGC 3 II 5 and IC 3973 were exhibited.

$H$. Bondi commented that he viewed this theory with mistrust. It was well-known that general relativity reduced to Newtonian theory when gravitational potentials and pressure/ density ratios were small. Edelen's theory did not satisfy this requirement, nor was there a Newtonian analogue to the discreteness. He suspected that incorrect boundary conditions had been applied to a system of hyperbolic equations, a procedure which could lead to extraordinary, but wholly invalid, results. G. C. McVittie questioned the relevance of the observed boundaries of galaxies that had been shown. These boundaries were the result of perspective produced by the relative positions of the galaxy and the observer. They therefore could not, except by accident, correspond to the calculated contours. Discussion followed.

The other speakers on the Evolution of Galaxies and the titles of their papers were the following:

E. Holmberg: 'The dependence of rate of evolution on mass-density'; Special reference was given to Dr Holmberg's recent results published in Uppsala astr. Obs. Medd., no. 148, 1964. G. Münch: 'Rotational velocities of the nuclear bulge of $\mathrm{M}_{3} \mathrm{I}$ ';

M. Burbidge: 'Non-circular motions in the central region of $\mathrm{M}_{3} \mathrm{I}$ ';

W. A. Baum (read by A. Sandage): 'Nature and evolution of spiral arms';

P. O. Lindblad: 'Dynamics of spiral arms';

$H$. Greyber: 'A galactic dipole model for spiral galaxies';

B. Lindblad: 'Circulation of matter in spiral arms';

E. L. Scott: 'The problem of the temporal sequence of morphological types';

$G$. de Vaucouleurs: 'Segregation of galaxy types in groups and clusters'. 